\title{
First Order Languages: Further Syntax and Semantics ${ }^{1}$
}

\author{
Marco B. Caminati ${ }^{2}$ \\ Mathematics Department "G.Castelnuovo" \\ Sapienza University of Rome \\ Piazzale Aldo Moro 5, 00185 Roma, Italy
}

\begin{abstract}
Summary. Third of a series of articles laying down the bases for classical first order model theory. Interpretation of a language in a universe set. Evaluation of a term in a universe. Truth evaluation of an atomic formula. Reassigning the value of a symbol in a given interpretation. Syntax and semantics of a non atomic formula are then defined concurrently (this point is explained in [16], 4.2.1). As a consequence, the evaluation of any w.f.f. string and the relation of logical implication are introduced. Depth of a formula. Definition of satisfaction and entailment (aka entailment or logical implication) relations, see [18] III.3.2 and III.4.1 respectively.
\end{abstract}

MML identifier: FOMODEL2, version: $\underline{7.11 .07 \quad 4.160 .1126}$

The terminology and notation used in this paper have been introduced in the following papers: [7], [1], [23], [6], [8], [17], [14], [15], [22], [9], [10], [11], [2], [21], [26], [24], [5], [3], [4], [12], [27], [28], [19], [20], [25], and [13].

For simplicity, we follow the rules: $m, n$ denote natural numbers, $m_{1}$ denotes an element of $\mathbb{N}, A, B, X, Y, Z, x, y$ denote sets, $S, S_{1}, S_{2}$ denote languages, $s$ denotes an element of $S, w, w_{1}, w_{2}$ denote strings of $S, U$ denotes a non empty set, $f, g$ denote functions, and $p, p_{2}$ denote finite sequences.

Let us consider $S$. Then TheNorSymbOf $S$ is an element of $S$.

Let $U$ be a non empty set. The functor $U$-deltaInterpreter yielding a function from $U^{2}$ into Boolean is defined by:

(Def. 1) $U$-deltaInterpreter $=\chi_{(\text {the concatenation of } U)^{\circ}\left(\operatorname{id}_{U^{1}}\right), U^{2}}$.

\footnotetext{
${ }^{1}$ The author wrote this paper as part of his $\mathrm{PhD}$ thesis research.

${ }^{2}$ I would like to thank Marco Pedicini for his encouragement and support.
} 
Let $X$ be a set. Then $\operatorname{id}_{X}$ is an equivalence relation of $X$.

Let $S$ be a language, let $U$ be a non empty set, and let $s$ be an of-atomicformula element of $S$. Interpreter of $s$ and $U$ is defined as follows:

(Def. 2)(i) It is a function from $U^{|\operatorname{ar} s|}$ into Boolean if $s$ is relational,

(ii) it is a function from $U^{|\operatorname{ar} s|}$ into $U$, otherwise.

Let us consider $S, U$ and let $s$ be an of-atomic-formula element of $S$. We see that the interpreter of $s$ and $U$ is a function from $U^{|a r s|}$ into $U \cup$ Boolean .

Let us consider $S, U$ and let $s$ be a termal element of $S$. One can verify that every interpreter of $s$ and $U$ is $U$-valued.

Let $S$ be a language. Note that every element of $S$ which is literal is also own.

Let us consider $S, U$. A function is called an interpreter of $S$ and $U$ if:

(Def. 3) For every own element $s$ of $S$ holds it $(s)$ is an interpreter of $s$ and $U$.

Let us consider $S, U, f$. We say that $f$ is $(S, U)$-interpreter-like if and only if:

(Def. 4) $\quad f$ is an interpreter of $S$ and $U$ and function yielding.

Let us consider $S$ and let $U$ be a non empty set. One can verify that every function which is $(S, U)$-interpreter-like is also function yielding.

Let us consider $S, U$ and let $s$ be an own element of $S$. Observe that every interpreter of $s$ and $U$ is non empty.

Let $S$ be a language and let $U$ be a non empty set. Note that there exists a function which is $(S, U)$-interpreter-like.

Let us consider $S, U$, let $I$ be an $(S, U)$-interpreter-like function, and let $s$ be an own element of $S$. Then $I(s)$ is an interpreter of $s$ and $U$.

Let $S$ be a language, let $U$ be a non empty set, let $I$ be an $(S, U)$-interpreterlike function, let $x$ be an own element of $S$, and let $f$ be an interpreter of $x$ and $U$. One can check that $I+\cdot(x \longmapsto f)$ is $(S, U)$-interpreter-like.

Let us consider $f, x, y$. The functor $(x, y)$ ReassignIn $f$ yields a function and is defined by:

(Def. 5) $\quad(x, y)$ ReassignIn $f=f+\cdot(x \longmapsto(\emptyset \longmapsto y))$.

Let $S$ be a language, let $U$ be a non empty set, let $I$ be an $(S, U)$-interpreterlike function, let $x$ be a literal element of $S$, and let $u$ be an element of $U$. One can verify that $(x, u)$ ReassignIn $I$ is $(S, U)$-interpreter-like.

Let $S$ be a language. One can check that AllSymbolsOf $S$ is non empty.

Let $Y$ be a set and let $X, Z$ be non empty sets. Observe that every function from $X$ into $Z^{Y}$ is function yielding.

Let $X, Y, Z$ be non empty sets. One can verify that there exists a function from $X$ into $Z^{Y}$ which is function yielding.

Let $f$ be a function yielding function and let $g$ be a function. The functor $[g, f]$ yields a function and is defined by: 
(Def. 6) $\operatorname{dom}[g, f]=\operatorname{dom} f$ and for every $x$ such that $x \in \operatorname{dom} f$ holds $[g, f](x)=$ $g \cdot f(x)$.

Let $f$ be an empty function and let $g$ be a function. One can verify that $[g, f]$ is empty.

Let $f$ be a function yielding function and let $g$ be a function. The functor $[f, g]$ yielding a function is defined as follows:

(Def. 7) $\operatorname{dom}[f, g]=\operatorname{dom} f \cap \operatorname{dom} g$ and for every set $x$ such that $x \in \operatorname{dom}[f, g]$ holds $[f, g](x)=f(x)(g(x))$.

Let $f$ be a function yielding function and let $g$ be an empty function. One can check that $[f, g]$ is empty.

Let $X$ be a finite sequence-membered set. Observe that every function which is $X$-valued is also function yielding.

Let $E, D$ be non empty sets, let $p$ be a $D$-valued finite sequence, and let $h$ be a function from $D$ into $E$. Note that $h \cdot p$ is len $p$-element.

Let $X, Y$ be non empty sets, let $f$ be a function from $X$ into $Y$, and let $p$ be an $X$-valued finite sequence. One can verify that $f \cdot p$ is finite sequence-like.

Let $E, D$ be non empty sets, let $n$ be a natural number, let $p$ be an $n$-element $D$-valued finite sequence, and let $h$ be a function from $D$ into $E$. Observe that $h \cdot p$ is $n$-element.

We now state the proposition

(1) For every 0-termal string $t_{0}$ of $S$ holds $t_{0}=\left\langle S\right.$-firstChar $\left.\left(t_{0}\right)\right\rangle$.

Let us consider $S$, let $U$ be a non empty set, let $u$ be an element of $U$, and let $I$ be an $(S, U)$-interpreter-like function. The functor $(I, u)$-TermEval yields a function from $\mathbb{N}$ into $U^{\text {AllTermsOf } S}$ and is defined as follows:

(Def. 8) $(I, u)$-TermEval(0) $=$ AllTermsOf $S \longmapsto u$ and for every $m_{1}$ holds $(I, u)-\operatorname{TermEval}\left(m_{1}+1\right)=\left[I \cdot S\right.$-firstChar, $\left[\left((I, u)-\operatorname{TermEval}\left(m_{1}\right)\right.\right.$ qua function), $S$-subTerms]].

Let us consider $S, U$, let $I$ be an $(S, U)$-interpreter-like function, and let $t$ be an element of AllTermsOf $S$. The functor $I$-TermEval $t$ yields an element of $U$ and is defined as follows:

(Def. 9) For every element $u_{1}$ of $U$ and for every $m_{1}$ such that $t \in$ $S$-termsOfMaxDepth $\left(m_{1}\right)$ holds $I$-TermEval $t=\left(I, u_{1}\right)$-TermEval $\left(m_{1}+\right.$ $1)(t)$.

Let us consider $S, U$ and let $I$ be an $(S, U)$-interpreter-like function. The functor $I$-TermEval yielding a function from AllTermsOf $S$ into $U$ is defined by:

(Def. 10) For every element $t$ of AllTermsOf $S$ holds $I$-TermEval $(t)=$ $I$-TermEval $t$.

Let us consider $S, U$ and let $I$ be an $(S, U)$-interpreter-like function. The functor $I===$ yielding a function is defined as follows:

(Def. 11) $I====I+\cdot($ TheEqSymbOf $S \longmapsto U$-deltaInterpreter). 
Let us consider $S, U$, let $I$ be an $(S, U)$-interpreter-like function, and let $x$ be a set. We say that $x$ is $I$-extension if and only if:

(Def. 12) $x=I===$.

Let us consider $S, U$ and let $I$ be an $(S, U)$-interpreter-like function. Note that $I===$ is $I$-extension and every set which is $I$-extension is also functionlike. Observe that there exists a function which is $I$-extension. Observe that $I===$ is $(S, U)$-interpreter-like.

Let $f$ be an $I$-extension function, and let $s$ be an of-atomic-formula element of $S$. Then $f(s)$ is an interpreter of $s$ and $U$.

Let $p_{1}$ be a 0 -w.f.f. string of $S$. The functor $I$-AtomicEval $p_{1}$ is defined as follows:

(Def. 13) $I$-AtomicEval $p_{1}=\left(I===\left(S\right.\right.$-firstChar $\left.\left.\left(p_{1}\right)\right)\right)\left(I\right.$-TermEval $\left.\cdot \operatorname{SubTerms~} p_{1}\right)$.

Let us consider $S, U$, let $I$ be an $(S, U)$-interpreter-like function, and let $p_{1}$ be a 0 -w.f.f. string of $S$. Then $I$-AtomicEval $p_{1}$ is an element of Boolean. Note that $I \uparrow$ OwnSymbolsOf $S$ is $\left(U^{*} \dot{\rightarrow}(U \cup\right.$ Boolean $\left.)\right)$-valued and $I \uparrow$ OwnSymbolsOf $S$ is $(S, U)$-interpreter-like.

Let us consider $S, U$ and let $I$ be an $(S, U)$-interpreter-like function. Observe that $I\lceil$ OwnSymbolsOf $S$ is total.

Let us consider $S, U$. The functor $U$-InterpretersOf $S$ is defined by:

(Def. 14) $U$-InterpretersOf $S=\left\{f \in\left(U^{*} \dot{\rightarrow}(U \cup \text { Boolean })\right)^{\text {OwnSymbolsOf } S: f \text { is }}\right.$ $(S, U)$-interpreter-like $\}$.

Let us consider $S, U$. Then $U$-InterpretersOf $S$ is a subset of $\left(U^{*} \rightarrow(U \cup\right.$ Boolean $))^{\text {OwnSymbolsOf } S}$. Observe that $U$-InterpretersOf $S$ is non empty. One can verify that every element of $U$-InterpretersOf $S$ is $(S, U)$-interpreter-like. The functor $S$-TruthEval $U$ yields a function from

$(U$-InterpretersOf $S) \times$ AtomicFormulasOf $S$ into Boolean and is defined by:

(Def. 15) For every element $I$ of $U$-InterpretersOf $S$ and for every element $p_{1}$ of AtomicFormulasOf $S$ holds $(S$-TruthEval $U)\left(I, p_{1}\right)=I$-AtomicEval $p_{1}$.

Let us consider $S, U$, let $I$ be an element of $U$-InterpretersOf $S$, let $f$ be a partial function from $(U$-InterpretersOf $S) \times\left((\text { AllSymbolsOf } S)^{*} \backslash\{\emptyset\}\right)$ to Boolean, and let $p_{1}$ be an element of (AllSymbolsOf $\left.S\right)^{*} \backslash\{\emptyset\}$. The functor $f$-ExFunctor $\left(I, p_{1}\right)$ yielding an element of Boolean is defined as follows:

(Def. 16) $f$-ExFunctor $\left(I, p_{1}\right)=\left\{\begin{array}{c}\text { true, } \\ \text { if there exists an element } u \text { of } U \text { and } \\ \text { there exists a literal element } v \text { of } S \text { such } \\ \text { that } p_{1}(1)=v \text { and } \\ f\left((v, u) \text { ReassignIn } I,\left(p_{1}\right)_{\downarrow 1}\right)=\text { true, } \\ \text { false, } \text { otherwise. }\end{array}\right.$

Let us consider $S, U$ and let $g$ be an element of $(U$-InterpretersOf $S) \times$ $\left((\text { AllSymbolsOf } S)^{*} \backslash\{\emptyset\}\right) \rightarrow$ Boolean . The functor ExIterator $g$ yields a partial function from $(U$-InterpretersOf $S) \times\left((\text { AllSymbolsOf } S)^{*} \backslash\{\emptyset\}\right)$ to Boolean and 
is defined by the conditions (Def. 17).

(Def. 17)(i) For every element $x$ of $U$-InterpretersOf $S$ and for every element $y$ of (AllSymbolsOf $S)^{*} \backslash\{\emptyset\}$ holds $\langle x, y\rangle \in$ dom ExIterator $g$ iff there exists a literal element $v$ of $S$ and there exists a string $w$ of $S$ such that $\langle x$, $w\rangle \in \operatorname{dom} g$ and $y=\langle v\rangle \frown w$, and

(ii) for every element $x$ of $U$-InterpretersOf $S$ and for every element $y$ of (AllSymbolsOf $S)^{*} \backslash\{\emptyset\}$ such that $\langle x, y\rangle \in \operatorname{dom}$ ExIterator $g$ holds $($ ExIterator $g)(x, y)=g$-ExFunctor $(x, y)$.

Let us consider $S, U$, let $f$ be a partial function from ( $U$-InterpretersOf $S) \times$ ((AllSymbolsOf $\left.S)^{*} \backslash\{\emptyset\}\right)$ to Boolean, let $I$ be an element of $U$-InterpretersOf $S$, and let $p_{1}$ be an element of (AllSymbolsOf $\left.S\right)^{*} \backslash\{\emptyset\}$.

The functor $f$-NorFunctor $\left(I, p_{1}\right)$ yielding an element of Boolean is defined by:

(Def. 18) $f$-NorFunctor $\left(I, p_{1}\right)=\left\{\begin{array}{l}\left\langle I, w_{1}\right\rangle \in \operatorname{dom} f \text { and } f\left(I, w_{1}\right)=\text { false } \\ \text { and } f\left(I, w_{2}\right)=\text { false and } \\ p_{1}=\langle\text { TheNorSymbOf } S\rangle \neg w_{1} \frown w_{2}, \\ \text { false, otherwise. }\end{array}\right.$

Let us consider $S, U$ and let $g$ be an element of $(U$-InterpretersOf $S) \times$ $\left((\text { AllSymbolsOf } S)^{*} \backslash\{\emptyset\}\right) \rightarrow$ Boolean . The functor NorIterator $g$ yielding a partial function from $(U$-InterpretersOf $S) \times\left((\text { AllSymbolsOf } S)^{*} \backslash\{\emptyset\}\right)$ to Boolean is defined by the conditions (Def. 19).

(Def. 19)(i) For every element $x$ of $U$-InterpretersOf $S$ and for every element $y$ of (AllSymbolsOf $S)^{*} \backslash\{\emptyset\}$ holds $\langle x, y\rangle \in$ dom NorIterator $g$ iff there exist elements $p_{3}, p_{4}$ of (AllSymbolsOf $\left.S\right)^{*} \backslash\{\emptyset\}$ such that $y=$ $\langle$ TheNorSymbOf $S\rangle\urcorner p_{3} \frown p_{4}$ and $\left\langle x, p_{3}\right\rangle,\left\langle x, p_{4}\right\rangle \in \operatorname{dom} g$, and

(ii) for every element $x$ of $U$-InterpretersOf $S$ and for every element $y$ of (AllSymbolsOf $S)^{*} \backslash\{\emptyset\}$ such that $\langle x, y\rangle \in$ dom NorIterator $g$ holds $($ NorIterator $g)(x, y)=g$-NorFunctor $(x, y)$.

Let us consider $S, U$. The functor $(S, U)$-TruthEval yields a function from $\mathbb{N}$ into $(U$-InterpretersOf $S) \times\left((\text { AllSymbolsOf } S)^{*} \backslash\{\emptyset\}\right) \dot{\rightarrow}$ Boolean and is defined as follows:

(Def. 20) $(S, U)$-TruthEval(0) $=S$-TruthEval $U$ and for every $m_{1}$ holds $(S, U)-\operatorname{TruthEval}\left(m_{1}+1\right)=\operatorname{ExIterator}(S, U)-\operatorname{TruthEval}\left(m_{1}\right)+\cdot$ NorIterator $(S, U)-\operatorname{TruthEval}\left(m_{1}\right)+\cdot(S, U)-\operatorname{TruthEval}\left(m_{1}\right)$.

Next we state the proposition

(2) For every $(S, U)$-interpreter-like function $I$ holds $I\lceil$ OwnSymbolsOf $S \in$ $U$-InterpretersOf $S$.

Let $S$ be a language, let $m$ be a natural number, and let $U$ be a non empty set. 
The functor $(S, U)$-TruthEval $m$ yielding an element of $(U$-InterpretersOf $S) \times$ $\left((\text { AllSymbolsOf } S)^{*} \backslash\{\emptyset\}\right) \rightarrow$ Boolean is defined as follows:

(Def. 21) For every $m_{1}$ such that $m=m_{1}$ holds $(S, U)$-TruthEval $m=$ $(S, U)$-TruthEval $\left(m_{1}\right)$.

Let us consider $S, U, m$ and let $I$ be an element of $U$-InterpretersOf $S$. The functor $(I, m)$-TruthEval yields an element of

$\left((\text { AllSymbolsOf } S)^{*} \backslash\{\emptyset\}\right) \rightarrow$ Boolean and is defined by:

(Def. 22) $(I, m)-$ TruthEval $=(\operatorname{curry}((S, U)-$ TruthEval $m))(I)$.

Let us consider $S, m$. The functor $S$-formulasOfMaxDepth $m$ yielding a subset of (AllSymbolsOf $S)^{*} \backslash\{\emptyset\}$ is defined as follows:

(Def. 23) For every non empty set $U$ and for every element $I$ of $U$-InterpretersOf $S$ and for every element $m_{1}$ of $\mathbb{N}$ such that $m=m_{1}$ holds $S$-formulasOfMaxDepth $m=\operatorname{dom}\left(\left(I, m_{1}\right)\right.$-TruthEval).

Let us consider $S, m, w$. We say that $w$ is $m$-w.f.f. if and only if:

(Def. 24) $w \in S$-formulasOfMaxDepth $m$.

Let us consider $S, w$. We say that $w$ is w.f.f. if and only if:

(Def. 25) There exists $m$ such that $w$ is $m$-w.f.f..

Let us consider $S$. Note that every string of $S$ which is 0-w.f.f. is also 0-w.f.f. and every string of $S$ which is 0 -w.f.f. is also 0-w.f.f.. Let us consider $m$. One can check that every string of $S$ which is $m$-w.f.f. is also w.f.f.. Let us consider $n$. One can check that every string of $S$ which is $m+0 \cdot n$-w.f.f. is also $m+n$-w.f.f..

Let us consider $S, m$. Observe that there exists a string of $S$ which is $m$ w.f.f.. Note that $S$-formulasOfMaxDepth $m$ is non empty. One can verify that there exists a string of $S$ which is w.f.f..

Let us consider $S, U$, let $I$ be an element of $U$-InterpretersOf $S$, and let $w$ be a w.f.f. string of $S$. The functor $I$-TruthEval $w$ yields an element of Boolean and is defined as follows:

(Def. 26) For every natural number $m$ such that $w$ is $m$-w.f.f. holds $I$-TruthEval $w=(I, m)-\operatorname{TruthEval}(w)$.

Let us consider $S$. The functor AllFormulas $S$ is defined by:

(Def. 27) AllFormulasOf $S=\left\{w ; w\right.$ ranges over strings of $S: \bigvee_{m} w$ is $m$-w.f.f. $\}$.

Let us consider $S$. One can check that AllFormulasOf $S$ is non empty.

For simplicity, we follow the rules: $u, u_{1}, u_{2}$ are elements of $U, t$ is a termal string of $S, I$ is an $(S, U)$-interpreter-like function, $l, l_{1}, l_{2}$ are literal elements of $S, m_{2}, n_{1}$ are non zero natural numbers, $p_{0}$ is a 0 -w.f.f. string of $S$, and $p_{5}$, $p_{1}, p_{3}, p_{4}$ are w.f.f. strings of $S$.

The following propositions are true:

(3) $(I, u)-\operatorname{TermEval}(m+1)(t)=I(S$-firstChar $(t))((I, u)-\operatorname{TermEval}(m)$. SubTerms $t)$ and if $t$ is 0-termal, then $(I, u)$-TermEval $(m+1)(t)=$ $I(S$-firstChar $(t))(\emptyset)$. 
(4) For every $m$-termal string $t$ of $S$ holds $\left(I, u_{1}\right)$-TermEval $(m+1)(t)=$ $\left(I, u_{2}\right)$-TermEval $(m+1+n)(t)$.

(5) $\operatorname{curry}((S, U)$-TruthEval $m)$ is a function from $U$-InterpretersOf $S$ into $\left((\text { AllSymbolsOf } S)^{*} \backslash\{\emptyset\}\right) \dot{\rightarrow}$ Boolean .

(6) $x \in X \cup Y \cup Z$ iff $x \in X$ or $x \in Y$ or $x \in Z$.

(7) $S$-formulasOfMaxDepth $0=$ AtomicFormulasOf $S$.

Let us consider $S, m$. Then $S$-formulasOfMaxDepth $m$ can be characterized by the condition:

(Def. 28) For every non empty set $U$ and for every element $I$ of $U$-InterpretersOf $S$ holds $S$-formulasOfMaxDepth $m=\operatorname{dom}((I, m)$-TruthEval).

Next we state the proposition

(8) $(S, U)$-TruthEval $m \in$ Boolean $^{(U \text {-InterpretersOf } S) \times(S \text {-formulasOfMaxDepth } m)}$ and

$(S, U)$-TruthEval $(m) \in$ Boolean $^{(U-\text { InterpretersOf } S) \times(S \text {-formulasOfMaxDepth } m)}$.

Let us consider $S, m$. The functor $m$-ExFormulasOf $S$ is defined by:

(Def. 29) $m$-ExFormulasOf $S=\left\{\langle v\rangle^{\frown} p_{1}: v\right.$ ranges over elements of LettersOf $S, p_{1}$ ranges over elements of $S$-formulasOfMaxDepth $m\}$.

The functor $m$-NorFormulasOf $S$ is defined as follows:

(Def. 30) $m$-NorFormulasOf $S=\left\{\langle\right.$ TheNorSymbOf $S\rangle \frown p_{3}{ }^{\frown} p_{4}: p_{3}$ ranges over elements of $S$-formulasOfMaxDepth $m, p_{4}$ ranges over elements of $S$-formulasOfMaxDepth $m\}$.

Let us consider $S$ and let $w_{1}, w_{2}$ be strings of $S$. Then $w_{1} \frown w_{2}$ is a string of $S$.

Let us consider $S, s$. Then $\langle s\rangle$ is a string of $S$.

One can prove the following two propositions:

(9) $S$-formulasOfMaxDepth $(m+1)=$ $(m$-ExFormulasOf $S) \cup(m$-NorFormulasOf $S) \cup(S$-formulasOfMaxDepth $m)$.

(10) AtomicFormulasOf $S$ is $S$-prefix.

Let us consider $S$. Note that AtomicFormulasOf $S$ is $S$-prefix. Observe that $S$-formulasOfMaxDepth 0 is $S$-prefix.

Let us consider $p_{1}$. The functor Depth $p_{1}$ yielding a natural number is defined by:

(Def. 31) $p_{1}$ is Depth $p_{1}$-w.f.f. and for every $n$ such that $p_{1}$ is $n$-w.f.f. holds Depth $p_{1} \leq n$.

Let us consider $S, m$ and let $p_{3}, p_{4}$ be $m$-w.f.f. strings of $S$. Note that $\langle$ TheNorSymbOf $S\rangle \frown p_{3} \frown p_{4}$ is $m+1$-w.f.f..

Let us consider $S, p_{3}, p_{4}$. Observe that $\langle\text { TheNorSymbOf } S\rangle^{\frown} p_{3}{ }^{\frown} p_{4}$ is w.f.f..

Let us consider $S, m$, let $p_{1}$ be an $m$-w.f.f. string of $S$, and let $v$ be a literal element of $S$. Note that $\langle v\rangle^{\frown} p_{1}$ is $m+1$-w.f.f.. 
Let us consider $S, l, p_{1}$. Note that $\langle l\rangle^{\frown} p_{1}$ is w.f.f..

Let us consider $S, w$ and let $s$ be a non relational element of $S$. One can check that $\langle s\rangle \frown w$ is non 0 -w.f.f..

Let us consider $S, w_{1}, w_{2}$ and let $s$ be a non relational element of $S$. Observe that $\langle s\rangle{ }^{\frown} w_{1}{ }^{\frown} w_{2}$ is non 0 -w.f.f.

Let us consider $S$. Observe that TheNorSymbOf $S$ is non relational.

Let us consider $S, w$. Observe that $\langle\text { TheNorSymbOf } S\rangle^{\frown} w$ is non 0-w.f.f..

Let us consider $S, l, w$. Note that $\langle l\rangle \frown w$ is non 0-w.f.f..

Let us consider $S, w$. We say that $w$ is exal if and only if:

(Def. 32) $S$-firstChar $(w)$ is literal.

Let us consider $S, w, l$. One can verify that $\langle l\rangle^{\frown} w$ is exal.

Let us consider $S, m_{2}$. Observe that there exists an $m_{2}$-w.f.f. string of $S$ which is exal.

Let us consider $S$. Note that every string of $S$ which is exal is also non 0 -w.f.f..

Let us consider $S, m_{2}$. One can check that there exists an exal $m_{2}$-w.f.f. string of $S$ which is non 0 -w.f.f..

Let us consider $S$. One can verify that there exists an exal w.f.f. string of $S$ which is non 0 -w.f.f..

Let us consider $S$ and let $p_{1}$ be a non 0-w.f.f. w.f.f. string of $S$. Note that Depth $p_{1}$ is non zero.

Let us consider $S$ and let $w$ be a non 0-w.f.f. w.f.f. string of $S$. Observe that $S$-firstChar $(w)$ is non relational.

Let us consider $S, m$. Observe that $S$-formulasOfMaxDepth $m$ is $S$-prefix. Then AllFormulasOf $S$ is a subset of (AllSymbolsOf $S)^{*} \backslash\{\emptyset\}$. Observe that every element of AllFormulasOf $S$ is w.f.f.. Note that AllFormulasOf $S$ is $S$-prefix.

We now state three propositions:

(11) dom NorIterator $((S, U)$-TruthEval $m)=$ $(U$-InterpretersOf $S) \times(m$-NorFormulasOf $S)$.

(12) dom ExIterator $((S, U)$-TruthEval $m)=$ $(U$-InterpretersOf $S) \times(m$-ExFormulasOf $S)$.

(13) $U$-deltaInterpreter ${ }^{-1}(\{1\})=\{\langle u, u\rangle: u$ ranges over elements of $U\}$.

Let us consider $S$. Then TheEqSymbOf $S$ is an element of $S$.

Let us consider $S$. One can verify that ar TheEqSymbOf $S+2$ is zero and $\mid \operatorname{ar}$ TheEqSymbOf $S \mid-2$ is zero.

We now state two propositions:

(14) Let $p_{1}$ be a 0 -w.f.f. string of $S$ and $I$ be an $(S, U)$-interpreter-like function. Then

(i) if $S$-firstChar $\left(p_{1}\right) \neq$ TheEqSymbOf $S$, then $I$-AtomicEval $p_{1}=$ $I\left(S\right.$-firstChar $\left.\left(p_{1}\right)\right)\left(I\right.$-TermEval $\cdot$ SubTerms $\left.p_{1}\right)$, and 
(ii) if $S$-firstChar $\left(p_{1}\right)=$ TheEqSymbOf $S$, then $I$-AtomicEval $p_{1}=$ $U$-deltaInterpreter $\left(I\right.$-TermEval $\cdot$ SubTerms $\left.p_{1}\right)$.

(15) Let $I$ be an $(S, U)$-interpreter-like function and $p_{1}$ be a 0 -w.f.f. string of $S$. If $S$-firstChar $\left(p_{1}\right)=$ TheEqSymbOf $S$, then $I$-AtomicEval $p_{1}=1$ iff $I$-TermEval $\left(\left(\operatorname{SubTerms} p_{1}\right)(1)\right)=I$-TermEval $\left(\left(\operatorname{SubTerms} p_{1}\right)(2)\right)$.

Let us consider $S, m$. One can check that $m$-ExFormulasOf $S$ is non empty. Note that $m$-NorFormulasOf $S$ is non empty. Then $m$-NorFormulasOf $S$ is a subset of (AllSymbolsOf $S)^{*} \backslash\{\emptyset\}$.

Let us consider $S$ and let $w$ be an exal string of $S$. One can verify that $S$-firstChar $(w)$ is literal.

Let us consider $S, m$. Observe that every element of $m$-NorFormulasOf $S$ is non exal. Then $m$-ExFormulasOf $S$ is a subset of (AllSymbolsOf $S)^{*} \backslash\{\emptyset\}$.

Let us consider $S, m$. One can check that every element of $m$-ExFormulasOf $S$ is exal.

Let us consider $S$. One can check that there exists an element of $S$ which is non literal.

Let us consider $S, w$ and let $s$ be a non literal element of $S$. Note that $\langle s\rangle^{\frown} w$ is non exal.

Let us consider $S, w_{1}, w_{2}$ and let $s$ be a non literal element of $S$. Observe that $\langle s\rangle \frown w_{1} \frown w_{2}$ is non exal.

Let us consider $S$. Note that TheNorSymbOf $S$ is non literal.

Next we state the proposition

(16) $p_{1} \in$ AllFormulasOf $S$.

Let us consider $S, m, w$. We introduce $w$ is $m$-non-w.f.f. as an antonym of $w$ is $m$-w.f.f..

Let us consider $m, S$. One can verify that every string of $S$ which is non $m$-w.f.f. is also $m$-non-w.f.f..

Let us consider $S, p_{3}, p_{4}$. Observe that $\langle$ TheNorSymbOf $\left.S\rangle\right\urcorner p_{3} \frown p_{4}$ is $\max \left(\operatorname{Depth} p_{3}\right.$, Depth $\left.p_{4}\right)$-non-w.f.f..

Let us consider $S, p_{1}, l$. Note that $\langle l\rangle^{\frown} p_{1}$ is Depth $p_{1}$-non-w.f.f..

Let us consider $S, p_{1}, l$. One can check that $\langle l\rangle^{\frown} p_{1}$ is $1+\operatorname{Depth} p_{1}$-w.f.f..

Let us consider $S, U$. Observe that every element of $U$-InterpretersOf $S$ is OwnSymbolsOf $S$-defined.

Let us consider $S, U$. Note that there exists an element of $U$-InterpretersOf $S$ which is OwnSymbolsOf $S$-defined.

Let us consider $S, U$. Note that every OwnSymbolsOf $S$-defined element of $U$-InterpretersOf $S$ is total.

Let us consider $S, U$, let $I$ be an element of $U$-InterpretersOf $S$, let $x$ be a literal element of $S$, and let $u$ be an element of $U$. Then $(x, u)$ ReassignIn $I$ is an element of $U$-InterpretersOf $S$.

In the sequel $I$ denotes an element of $U$-InterpretersOf $S$. 
Let us consider $S, w$. The functor xnot $w$ yields a string of $S$ and is defined as follows:

(Def. 33) $\quad \operatorname{xnot} w=\langle\text { TheNorSymbOf } S\rangle^{\frown} w^{\frown} w$.

Let us consider $S, m$ and let $p_{1}$ be an $m$-w.f.f. string of $S$. Observe that $\operatorname{xnot} p_{1}$ is $m+1$-w.f.f..

Let us consider $S, p_{1}$. Note that $\operatorname{xnot} p_{1}$ is w.f.f..

Let us consider $S$. One can verify that TheEqSymbOf $S$ is non own.

Let us consider $S, X$. We say that $X$ is $S$-mincover if and only if:

(Def. 34) For every $p_{1}$ holds $p_{1} \in X$ iff xnot $p_{1} \notin X$.

One can prove the following propositions:

(17) $\operatorname{Depth}\left(\langle\text { TheNorSymbOf } S\rangle^{\frown} p_{3} \frown p_{4}\right)=1+\max \left(\operatorname{Depth} p_{3}\right.$, Depth $\left.p_{4}\right)$ and $\operatorname{Depth}\left(\langle l\rangle \frown p_{3}\right)=\operatorname{Depth} p_{3}+1$.

(18) If Depth $p_{1}=m+1$, then $p_{1}$ is exal iff $p_{1} \in m$-ExFormulasOf $S$ and $p_{1}$ is non exal iff $p_{1} \in m$-NorFormulasOf $S$.

(19) $I$-TruthEval $\langle l\rangle \frown p_{1}=$ true iff there exists $u$ such that

$((l, u)$ ReassignIn $I)$-TruthEval $p_{1}=1$ and $I$-TruthEval $\langle\text { TheNorSymbOf } S\rangle^{\wedge}$ $p_{3}{ }^{\frown} p_{4}=$ true iff $I$-TruthEval $p_{3}=$ false and $I$-TruthEval $p_{4}=$ false.

In the sequel $I$ denotes an $(S, U)$-interpreter-like function.

One can prove the following two propositions:

(20) $(I, u)$-TermEval $(m+1)\lceil S$-termsOfMaxDepth $(m)=$ $I$-TermEval $\lceil S$-termsOfMaxDepth $(m)$.

(21) $I$-TermEval $(t)=I(S$-firstChar $(t))(I$-TermEval $\cdot$ SubTerms $t)$.

Let us consider $S, p_{1}$. The functor SubWffsOf $p_{1}$ is defined as follows:

(Def. 35)(i) There exist $p_{3}, p$ such that $p$ is AllSymbolsOf $S$-valued and SubWffsOf $p_{1}=\left\langle p_{3}, p\right\rangle$ and $p_{1}=\left\langle S \text {-firstChar }\left(p_{1}\right)\right\rangle^{\frown} p_{3} \frown p$ if $p_{1}$ is non 0 -w.f.f.,

(ii) SubWffsOf $p_{1}=\left\langle p_{1}, \emptyset\right\rangle$, otherwise.

Let us consider $S, p_{1}$. The functor head $p_{1}$ yields a w.f.f. string of $S$ and is defined as follows:

(Def. 36) head $p_{1}=\left(\text { SubWffsOf } p_{1}\right)_{1}$.

The functor tail $p_{1}$ yields an element of (AllSymbolsOf $\left.S\right)^{*}$ and is defined by:

(Def. 37) tail $p_{1}=\left(\text { SubWffsOf } p_{1}\right)_{2}$.

Let us consider $S, m$. One can verify that $(S$-formulasOfMaxDepth $m) \backslash$ AllFormulas Of $S$ is empty.

Let us consider $S$. Observe that AtomicFormulasOf $S \backslash$ AllFormulasOf $S$ is empty.

We now state two propositions:

(22) $\left.\operatorname{Depth}(\langle l\rangle\urcorner p_{3}\right)>\operatorname{Depth} p_{3}$ and $\operatorname{Depth}\left(\langle\text { TheNorSymbOf } S\rangle^{\frown} p_{3} \frown p_{4}\right)>$ $\operatorname{Depth} p_{3}$ and Depth $\left(\langle\right.$ TheNorSymbOf $\left.S\rangle \frown p_{3} \frown p_{4}\right)>\operatorname{Depth} p_{4}$. 
(23) If $p_{1}$ is not 0 -w.f.f., then $\left.p_{1}=\langle x\rangle\right\rangle^{\frown} p_{4}{ }^{\frown} p_{2}$ iff $x=S$-firstChar $\left(p_{1}\right)$ and $p_{4}=$ head $p_{1}$ and $p_{2}=$ tail $p_{1}$.

Let us consider $S, m_{2}$. Observe that there exists a non 0 -w.f.f. $m_{2}$-w.f.f. string of $S$ which is non exal.

Let us consider $S$ and let $p_{1}$ be an exal w.f.f. string of $S$. One can verify that tail $p_{1}$ is empty.

Let us consider $S$ and let $p_{1}$ be a non exal non 0-w.f.f. w.f.f. string of $S$. Then tail $p_{1}$ is a w.f.f. string of $S$.

Let us consider $S$ and let $p_{1}$ be a non exal non 0-w.f.f. w.f.f. string of $S$. One can check that tail $p_{1}$ is w.f.f..

Let us consider $S$ and let $p_{1}$ be a non 0-w.f.f. non exal w.f.f. string of $S$. One can verify that $S$-firstChar $\left(p_{1}\right)-$ TheNorSymbOf $S$ is empty.

Let us consider $m, S$ and let $p_{1}$ be an $m+1$-w.f.f. string of $S$. Note that head $p_{1}$ is $m$-w.f.f..

Let us consider $m, S$ and let $p_{1}$ be an $m+1$-w.f.f. non exal non 0 -w.f.f. string of $S$. Observe that tail $p_{1}$ is $m$-w.f.f..

One can prove the following proposition

(24) For every element $I$ of $U$-InterpretersOf $S$ holds $(I, m)$-TruthEval $\in$ Boolean $^{S \text {-formulasOfMaxDepth } m}$.

Let us consider $S$. One can check that there exists an of-atomic-formula element of $S$ which is non literal.

One can prove the following proposition

(25) If $l_{2} \notin \operatorname{rng} p$, then $\left(\left(l_{2}, u\right) \operatorname{ReassignIn} I\right)-\operatorname{TermEval}(p)=I$-TermEval $(p)$.

Let us consider $X, S, s$. We say that $s$ is $X$-occurring if and only if:

(Def. 38) $s \in$ SymbolsOf $\left(\left((\text { AllSymbolsOf } S)^{*} \backslash\{\emptyset\}\right) \cap X\right)$.

Let us consider $S, s$ and let us consider $X$. We say that $X$ is $s$-containing if and only if:

(Def. 39) $s \in$ SymbolsOf((AllSymbolsOf $\left.S)^{*} \backslash\{\emptyset\} \cap X\right)$.

Let us consider $X, S, s$. We introduce $s$ is $X$-absent as an antonym of $s$ is $X$-occurring.

Let us consider $S, s, X$. We introduce $X$ is $s$-free as an antonym of $X$ is $s$-containing.

Let $X$ be a finite set and let us consider $S$. Observe that there exists a literal element of $S$ which is $X$-absent.

Let us consider $S, t$. Note that $\operatorname{rng} t \cap$ LettersOf $S$ is non empty.

Let us consider $S, p_{1}$. One can verify that $\operatorname{rng} p_{1} \cap$ LettersOf $S$ is non empty.

Let us consider $B, S$ and let $A$ be a subset of $B$. Note that every element of $S$ which is $A$-occurring is also $B$-occurring.

Let us consider $A, B, S$. Observe that every element of $S$ which is $A$ null $B$ absent is also $A \cap B$-absent. 
Let $F$ be a finite set and let us consider $A, S$. Note that every $F$-absent element of $S$ which is $A$-absent is also $A \cup F$-absent.

Let us consider $S, U$ and let $I$ be an $(S, U)$-interpreter-like function. One can check that OwnSymbolsOf $S \backslash \operatorname{dom} I$ is empty.

One can prove the following proposition

(26) There exists $u$ such that $u=I(l)(\emptyset)$ and $(l, u)$ ReassignIn $I=I$.

Let us consider $S, X$. We say that $X$ is $S$-covering if and only if:

(Def. 40) For every $p_{1}$ holds $p_{1} \in X$ or $\operatorname{xnot} p_{1} \in X$.

Let us consider $S$. One can check that every set which is $S$-mincover is also $S$-covering.

Let us consider $U$, let $p_{1}$ be a non 0 -w.f.f. non exal w.f.f. string of $S$, and let $I$ be an element of $U$-InterpretersOf $S$.

One can verify that $\left(I\right.$-TruthEval $\left.p_{1}\right)-\left(\left(I\right.\right.$-TruthEval head $\left.p_{1}\right)$ 'nor'

$\left(I\right.$-TruthEval tail $\left.\left.p_{1}\right)\right)$ is empty.

The functor ExFormulasOf $S$ yielding a subset of (AllSymbolsOf $S)^{*} \backslash\{\emptyset\}$ is defined by:

(Def. 41) ExFormulasOf $S=\left\{p_{1} ; p_{1}\right.$ ranges over strings of $S: p_{1}$ is w.f.f. $\wedge p_{1}$ is exal\}.

Let us consider $S$. Note that ExFormulasOf $S$ is non empty.

Let us consider $S$. One can check that every element of ExFormulasOf $S$ is exal and w.f.f..

Let us consider $S$. Note that every element of ExFormulasOf $S$ is w.f.f..

Let us consider $S$. Observe that every element of ExFormulasOf $S$ is exal.

Let us consider $S$. Observe that ExFormulasOf $S \backslash$ AllFormulasOf $S$ is empty.

Let us consider $U, S_{1}$ and let $S_{2}$ be an $S_{1}$-extending language. Note that every function which is $\left(S_{2}, U\right)$-interpreter-like is also $\left(S_{1}, U\right)$-interpreter-like.

Let us consider $U, S_{1}$, let $S_{2}$ be an $S_{1}$-extending language, and let $I$ be an $\left(S_{2}, U\right)$-interpreter-like function. Observe that $I\left\lceil\right.$ OwnSymbolsOf $S_{1}$ is $\left(S_{1}, U\right)$ interpreter-like.

Let us consider $U, S_{1}$, let $S_{2}$ be an $S_{1}$-extending language, let $I_{1}$ be an element of $U$-InterpretersOf $S_{1}$, and let $I_{2}$ be an $\left(S_{2}, U\right)$-interpreter-like function. Note that $I_{2}+\cdot I_{1}$ is $\left(S_{2}, U\right)$-interpreter-like.

Let us consider $U, S$, let $I$ be an element of $U$-InterpretersOf $S$, and let us consider $X$. We say that $X$ is $I$-satisfied if and only if:

(Def. 42) For every $p_{1}$ such that $p_{1} \in X$ holds $I$-TruthEval $p_{1}=1$.

Let us consider $S, U, X$ and let $I$ be an element of $U$-InterpretersOf $S$. We say that $I$ satisfies $X$ if and only if:

(Def. 43) $X$ is $I$-satisfied.

Let us consider $U, S$, let $e$ be an empty set, and let $I$ be an element of $U$-InterpretersOf $S$. Observe that $e$ null $I$ is $I$-satisfied. 
Let us consider $X, U, S$ and let $I$ be an element of $U$-InterpretersOf $S$. Observe that there exists a subset of $X$ which is $I$-satisfied.

Let us consider $U, S$ and let $I$ be an element of $U$-InterpretersOf $S$. One can check that there exists a set which is $I$-satisfied.

Let us consider $U, S$, let $I$ be an element of $U$-InterpretersOf $S$, and let $X$ be an $I$-satisfied set. One can check that every subset of $X$ is $I$-satisfied.

Let us consider $U, S$, let $I$ be an element of $U$-InterpretersOf $S$, and let $X$, $Y$ be $I$-satisfied sets. One can verify that $X \cup Y$ is $I$-satisfied.

Let us consider $U, S$, let $I$ be an element of $U$-InterpretersOf $S$, and let $X$ be an $I$-satisfied set. Observe that $I$ null $X$ satisfies $X$.

Let us consider $S, X$. We say that $X$ is $S$-correct if and only if the condition (Def. 44) is satisfied.

(Def. 44) Let $U$ be a non empty set, $I$ be an element of $U$-InterpretersOf $S, x$ be an $I$-satisfied set, and given $p_{1}$. If $\left\langle x, p_{1}\right\rangle \in X$, then $I$-TruthEval $p_{1}=1$.

Let us consider $S$. Note that $\emptyset$ null $S$ is $S$-correct.

Let us consider $S, X$. Observe that there exists a subset of $X$ which is $S$-correct.

Next we state two propositions:

(27) For every element $I$ of $U$-InterpretersOf $S$ holds $I$-TruthEval $p_{1}=1$ iff $\left\{p_{1}\right\}$ is $I$-satisfied.

(28) $s$ is $\{w\}$-occurring iff $s \in \operatorname{rng} w$.

Let us consider $U, S$, let us consider $p_{3}, p_{4}$, and let $I$ be an element of $U$-InterpretersOf $S$. Observe that $\left(I\right.$-TruthEval $\left.\langle\text { TheNorSymbOf } S\rangle^{\frown} p_{3} \frown p_{4}\right) \dot{-}$

$\left(\left(I\right.\right.$-TruthEval $\left.p_{3}\right)$ 'nor' $\left(I\right.$-TruthEval $\left.\left.p_{4}\right)\right)$ is empty.

Let us consider $S, p_{1}, U$ and let $I$ be an element of $U$-InterpretersOf $S$. Note that $\left(I\right.$-TruthEval xnot $\left.p_{1}\right)-\neg\left(I\right.$-TruthEval $\left.p_{1}\right)$ is empty.

Let us consider $X, S, p_{1}$. We say that $p_{1}$ is $X$-implied if and only if:

(Def. 45) For every non empty set $U$ and for every element $I$ of $U$-InterpretersOf $S$ such that $X$ is $I$-satisfied holds $I$-TruthEval $p_{1}=1$.

\section{REFERENCES}

[1] Grzegorz Bancerek. Cardinal numbers. Formalized Mathematics, 1(2):377-382, 1990.

[2] Grzegorz Bancerek. Curried and uncurried functions. Formalized Mathematics, 1(3):537$541,1990$.

[3] Grzegorz Bancerek. The fundamental properties of natural numbers. Formalized Mathematics, 1(1):41-46, 1990.

[4] Grzegorz Bancerek. The ordinal numbers. Formalized Mathematics, 1(1):91-96, 1990.

[5] Grzegorz Bancerek. Monoids. Formalized Mathematics, 3(2):213-225, 1992.

[6] Grzegorz Bancerek and Krzysztof Hryniewiecki. Segments of natural numbers and finite sequences. Formalized Mathematics, 1(1):107-114, 1990.

[7] Czesław Byliński. Binary operations. Formalized Mathematics, 1(1):175-180, 1990.

[8] Czesław Byliński. Finite sequences and tuples of elements of a non-empty sets. Formalized Mathematics, 1(3):529-536, 1990. 
[9] Czesław Byliński. Functions and their basic properties. Formalized Mathematics, 1(1):5565, 1990.

[10] Czesław Byliński. Functions from a set to a set. Formalized Mathematics, 1(1):153-164, 1990.

[11] Czesław Byliński. The modification of a function by a function and the iteration of the composition of a function. Formalized Mathematics, 1(3):521-527, 1990.

[12] Czesław Byliński. Partial functions. Formalized Mathematics, 1(2):357-367, 1990.

[13] Czesław Byliński. Some basic properties of sets. Formalized Mathematics, 1(1):47-53, 1990.

[14] Marco B. Caminati. Preliminaries to classical first order model theory. Formalized Mathematics, 19(3):155-167, 2011, doi: 10.2478/v10037-011-0025-2.

[15] Marco B. Caminati. Definition of first order language with arbitrary alphabet. Syntax of terms, atomic formulas and their subterms. Formalized Mathematics, 19(3):169-178, 2011, doi: 10.2478/v10037-011-0026-1.

[16] M.B. Caminati. Basic first-order model theory in Mizar. Journal of Formalized Reasoning, 3(1):49-77, 2010.

[17] Agata Darmochwał. Finite sets. Formalized Mathematics, 1(1):165-167, 1990.

[18] H.D. Ebbinghaus, J. Flum, and W. Thomas. Mathematical logic. Springer, 1994.

[19] Jarosław Kotowicz. Functions and finite sequences of real numbers. Formalized Mathematics, 3(2):275-278, 1992.

[20] Jarosław Kotowicz and Yuji Sakai. Properties of partial functions from a domain to the set of real numbers. Formalized Mathematics, 3(2):279-288, 1992.

[21] Rafał Kwiatek and Grzegorz Zwara. The divisibility of integers and integer relative primes. Formalized Mathematics, 1(5):829-832, 1990.

[22] Andrzej Trybulec. Binary operations applied to functions. Formalized Mathematics, 1(2):329-334, 1990.

[23] Andrzej Trybulec. Domains and their Cartesian products. Formalized Mathematics, 1(1):115-122, 1990.

[24] Andrzej Trybulec. Tuples, projections and Cartesian products. Formalized Mathematics, 1(1):97-105, 1990.

[25] Zinaida Trybulec. Properties of subsets. Formalized Mathematics, 1(1):67-71, 1990.

[26] Edmund Woronowicz. Many-argument relations. Formalized Mathematics, 1(4):733-737, 1990.

[27] Edmund Woronowicz. Relations and their basic properties. Formalized Mathematics, 1(1):73-83, 1990.

[28] Edmund Woronowicz. Relations defined on sets. Formalized Mathematics, 1(1):181-186, 1990. 